\title{
MicroRNA- 126: a promising biomarker for angiogenesis of diabetic wounds treated with negative pressure wound therapy
}

This article was published in the following Dove Press journal: Diabetes, Metabolic Syndrome and Obesity: Targets and Therapy

\author{
Dong Zhang \\ Zonghuan Li \\ Zheng Wang \\ Fanwei Zeng \\ Weidong Xiao \\ Aixi Yu
}

Department of Orthopedics, Zhongnan Hospital of Wuhan University, Wuhan, Hubei 43007I, People's Republic of China
Background: Negative pressure wound therapy represents an effective therapy to treat nonhealing diabetic wounds by promoting angiogenesis, of which the mechanism hasn't been investigated thoroughly. Growing evidence suggests that miRNAs hold great potential to be clinical biomarkers, and miR-126 is an essential angiogenesis regulator in diabetic wound repair.

Purpose: Our study aims to explore the effect of NPWT on the expression of miR-126 in the wound tissue and plasma of diabetic rat models and the association between circulating miR-126 and two quantitative indexes of angiogenesis.

Methods: Full-thickness excisional wounds were created on the back of diabetic rats. Measure the wound closure and collect the wound tissue and blood for H\&E, immunohistochemistry, Western blot and RT-PCR. Here we demonstrated that significantly increased capillary density and arteriolar density in the NPWT group at each specified time-point.

Results: In the NPWT group, miR-126 expression was significantly increased on days 3, 5, 7 , and $9(P<0.05)$. Furthermore, statistically significant increases in VEGF mRNA and protein expression and p-ERK expression, as well as decreased SPRED1 expression, were noted upon treatment with NPWT on day 9. Our data revealed that miR-126 expression in the wound and plasma was significantly associated $(P<0.05)$. Moreover, a positive correlation was also detected between increased levels of circulating miR-126 and arteriolar density, as well as capillary density $(P<0.05)$.

Conclusion: The study suggested that miR-126 was upregulated by NPWT and could represent a promising monitoring tool for angiogenesis in diabetic wounds treated with NPWT.

Keywords: microRNA-126, negative-pressure wound therapy, diabetic wound healing

\section{Introduction}

Recent data indicate that the global prevalence of diabetes mellitus will likely reach up to 600 million by 2,035 , and this prevalence will impose serious personal and societal healthcare burdens worldwide. ${ }^{1}$ A nonhealing chronic wound is one of the most serious diabetic complications resulting in pain, suffering and poor quality life for people with diabetes. In contrast to normal wound healing, diabetic wounds, particularly those that involve the feet, are commonly associated with inadequate local angiogenesis that fails to supply sufficient oxygen and nutrients for wound repair. ${ }^{2}$ Moreover, the growth of new blood vessels is essential for granulation tissue formation, which is a crucial process of wound healing. ${ }^{3-5}$ Thus, it is imperative to identify efficient therapies to
Correspondence: Weidong Xiao; Aixi Yu Department of Orthopedics, Zhongnan Hospital of Wuhan University, No. 169, Donghu Road, Wuchang district, Wuhan, Hubei 43007I, People's Republic of China Tel +86 I 8707182868

+86 I 3507187489

Email 1539562641@qq.com;

yuaixi@whu.edu.cn 
increase angiogenesis and promote wound healing. Among all types of wound therapies, NPWT is an effective and safe option to generate a well-vascularized diabetic wound. NPWT consists of an adhesive dressing and a negative pressure generator, which are connected by a tube. The therapy has been widely used to accelerate the healing of various wounds for decades of years. ${ }^{6}$ In addition to keeping the wound moist, the removal of harmful substances and the induction of perfusion changes, wound healing is facilitated by the overexpression of vascular endothelial growth factor (VEGF). ${ }^{7}$ Within the field of various factors participating in the regulation of wound angiogenesis, VEGF is considered to play an indispensable role. ${ }^{8}$ VEGF also aids in wound healing by facilitating the formation of the granulation tissue. ${ }^{9}$ However, the underlying molecular mechanism regarding how NPWT influences VEGF expression remains unclear.

Although NPWT exhibits excellent performance in promoting wound healing by participating in angiogenesis, there are sporadic clinical cases involving impaired angiogenic response and ability to fight infections in the wound area that exhibit failing angiogenesis. ${ }^{10}$ Given that typical NPWT foam dressing is expensive and its adherence to the wound bed can cause acute pain and slow healing and recovery during dressing changes, it limits the possibility of frequent inspection and changes. Thus, the judgment is exclusively based on the clinical experience of the operating surgeon. Thus, it is clinically significant to identify some promising biomarkers isolated from peripheral blood that exhibit relevance in evaluating wound angiogenesis with NPWT and can guide the time for discontinuation to realize optimal costeffectiveness of the therapy in the future.

MicroRNAs (miRNAs) are highly conserved, nonprotein coding that are only 21-23 nucleotides long. MicroRNAs mainly match with diverse mRNAs in a $3^{\prime}$ noncoding region to regulate gene expression, which promotes mRNA degradation and subsequently inhibits protein translation. ${ }^{11,12}$ miRNA studies revealed that they control various cellular and pathological processes, such as proliferation, immunity, and metabolism. Recently, mounting studies have demonstrated that several specific miRNAs are critically involved in wound angiogenesis as angiogenic regulators and present in the circulation in a stable form both in humans and animals.13-18 Among them, miR-126, which is the only miRNA reported to be specifically expressed in endothelial cells, has been proposed as a significant angiogenic regulator and biomarker in various diseases. Specifically, in type 1 and 2 diabetes, miR-126 was strongly associated with micro/ macrovascular complications. miR-126 has been identified as a biological target and holds potential for use as a diagnostic and therapeutic tool. ${ }^{18-20}$ Some reports demonstrated that diabetic microangiopathy including diabetic wounds obtained a better curative effect via increasing the expression of miR-126 directly or indirectly. ${ }^{21,22}$ More importantly, miR-126 can increase the expression of VEGF by inhibiting negative regulators of the VEGF receptor pathway, including Sprouty-related EVH1 domain containing protein 1 (SPRED1) and phosphoinositol-3 kinase regulatory subunit 2 (PIK3R2). ${ }^{16}$ Moreover, reduction or deletion of miR-126 affects vascular integrity and causes defects in endothelial progenitor cell-mediated functional vascular properties. ${ }^{23}$

Although it has long been realized that miR-126 is a strong participant in multiple diabetic complications, there are no studies researching the effect of NPWT on miR-126 expression in diabetic wounds. Therefore, the aim of this study was to determine whether miR-126 is involved in angiogenesis in diabetic wounds treated with NPWT in both wound tissue and circulation. In addition, we sought to determine whether miR-126 could be used as a biomarker of the response to therapy by exploring the association between circulating miR-126 and blood vessel density.

\section{Materials and methods}

\section{Ethics statement}

All experiments were performed in compliance with the guidelines of the Institutional Animal Care and Use Committee (IACUC) of Wuhan University. The experimental protocol was approved by the Committee on the Ethics of Animal Experiments of Wuhan University, Wuhan, China (Approval number:2,018,088). All efforts were made to minimize animal suffering.

\section{Establishment of a diabetic wound healing model and grouping}

We purchased male Sprague-Dawley (SD) rats at age 8 weeks and weighting 260-300 g from the laboratory Animal Center of Wuhan University (China). After 3 days of adjusting to the new environment, all rats were induced by intraperitoneal injections of STZ $(60 \mathrm{mg} / \mathrm{kg}$ in citrate buffer with $\mathrm{pH} 4.5$, Sigma, USA), and then given free access to food and water. A blood sample via the tail vein was assessed using a glucometer. The blood glucose of rats was monitored every day before the wound surgery. Three days after injection,only rats with blood glucose greater than $300 \mathrm{mg} / \mathrm{dl}(16.7 \mathrm{mmol} / \mathrm{L})$ were considered diabetic and included in further step. During all the period 
of the experiment, those with levels below $180 \mathrm{mg} / \mathrm{dl}$ were considered non-diabetic and between $180 \mathrm{mg} / \mathrm{dl}$ and $300 \mathrm{mg} / \mathrm{dl}$ were excluded from the study. In addition, we measured the changes of the weight, water intake and urine output before the injection (baseline) and at 3 days post injection, which is the typical symptoms of diabetes mellitus, the results is shown on the Figure S1.

Thirty diabetic rats were randomly distributed into two group: the NPWT group and the control group (n=15/each group). All rats were placed under general anesthesia with ketamine hydrochloride ( $60 \mathrm{mg} / \mathrm{kg}$ body weight) before surgery. After the backs of the rats were shaved and disinfected, a full-thickness excisional wound (20 $\mathrm{mm}$ in diameter), including the panniculus carnosus, was created on the back of the rats. For the NPWT group, the wound was covered with a medical foam (VSD Medical Technology Inc., Wuhan, China), and constant $125 \mathrm{mmHg}$ suction was produced by a Vacuum-assisted Closure Device (VSD Medical Technology Inc., Wuhan, China), the appliance and the foam are shown on the Figure S2. For the control group, moist gauze dressings were placed on the wound. Dressings were changed every 3 days in two groups. The blood glucose of rats was measured at day $0,3,5,7,9$ after surgery.

\section{Measurement of wound closure and collection of wound tissue and blood}

Three animals were taken from each group and sacrificed for photographing wound and collecting samples, including wound tissue and blood at day $0,3,5,7$, and 9 postsurgery. The wound area was calculated using Image Pro Plus 6.0(IPP 6.0) software (media cybernetics, USA). The unhealed rate was calculated by comparing the unhealed wound area to the original wound area. Healing rates (\%) $=($ wound area on day $0-$ wound area on day " $X ") /($ wound area on day 0 ) $\times 100 \%$. Fresh granulation tissue from the center of the wound was obtained and subdivided into two portions. One section was fixed in 4\% paraformaldehyde and embedded in paraffin for hematoxylin-eosin (H\&E) staining and immunohistochemistry. The other was stored at $-80{ }^{\circ} \mathrm{C}$ for miRNA expression by RT-PCR and protein analysis by Western blot assay. Blood $(2 \mathrm{~mL})$ was collected into a $0.5-\mathrm{mL} \mathrm{K}_{2}$-EDTA tube from the lateral tail vein and then mixed well with EDTA by inverting the tube $10 \times 180^{\circ}$ and back. After the first step, we centrifuged the sample at $1,300 \times \mathrm{g}, 10 \mathrm{~min}$, and $4{ }^{\circ} \mathrm{C}$ to obtain the plasma $(50-200 \mu \mathrm{L})$, which was stored at -70 to $-80{ }^{\circ} \mathrm{C}$ until processing.

\section{Immunohistochemical analysis}

Immunohistochemistry was performed to assess wound angiogenesis on days $0,3,5,7$ and 9 . The wound tissue was embedded in paraffin and cut into $5 \mu \mathrm{m}$ slices. Quantification of capillary density and arteriolar density were assessed by immunohistochemical staining with CD31, which is regarded as an endothelial cell marker, and $\alpha$-SMA, which served as a smooth muscle cell marker. Furthermore, 5 randomly selected areas from 3 sections from different animals were double blindly counted three times with magnification x200. The number of CD31-positive cells per square millimeter was used to quantify the capillary density. Similarly, arteriolar density was expressed as the number of $\alpha$-SMA-positive cells per square millimeter.

\section{Real-time quantitative PCR}

Total RNA was extracted from the wound tissue and plasma using TRIzol Reagent (InvitrogenTM, Carlsbad, CA) following the manufacturer's instructions. cDNA was synthesized using the PrimeScript II First Strand cDNA Synthesis Kit in accordance with the manufacturer's protocol (Takara Bio, Japan). Briefly, conditions of the reaction were as follows: an initial step of denaturation for $1 \mathrm{~min}$ at $95^{\circ} \mathrm{C}$ followed by 40 cycles of $95^{\circ} \mathrm{C}$ for $30 \mathrm{~s}, 60^{\circ} \mathrm{C}$ for $20 \mathrm{~s}$, and $70^{\circ} \mathrm{C}$ for $1 \mathrm{~s}$. The relative expression level of miRNA-126 was normalized to U6 small nuclear RNA expression, and mRNA expression was normalized to the housekeeping gene GAPDH. All RNAs were computed using the $2^{\Delta \Delta C T}$ method. The analyses for miR-126 were operated at each special time (day 0, day 3 , day 5, day 7, and day 9 post-surgery), and analyses for SPRED1, PI3KR2 and VEGF were performed on day 9. The primers used for qRT-PCR are listed in Table 1.

\section{Western blotting analysis}

Total protein was isolated from the wound tissue obtained at indicated times with RIPA (Aspen Inc.) following the manufacturer's instruction. The protein concentrations were determined using the BCA (Aspen Inc.). Equal amounts of protein from each tissue were separated by SDS-PAGE (Aspen Inc.), transferred to a PVDF membrane, and incubated with $5 \%$ milk for $1 \mathrm{~h}$ at room temperature. The membranes were incubated overnight at $4{ }^{\circ} \mathrm{C}$ with rabbit anti-phosphorylated-ERK (1:1,000; Cell Signaling Technology), rabbit anti-ERK (1:2,000; Cell Signaling Technology), rabbit anti-phosphorylated-AKT (1:1,000; Cell Signaling Technology), rabbit anti-AKT (2:1,000; Cell Signaling Technology), rabbit anti- 
Table I Primer sequences for reverse transcription-quantitative polymerase chain reaction

\begin{tabular}{|l|l|l|l|}
\hline Genes & Forward & Reverse & GenBank Accession numbers \\
\hline VEGF & ATCTTCAAGCCGTCCTGTGTG & AGGTTTGATCCGCATGATCTG & NM_00III0333.2 \\
SPREDI & GCTTTTGATCGAGGCATTCG & ATGGTATCTGGCTCACTTGGCT & NM_00I047089.I \\
PIK3R2 & TGCCACCCAAACCCTCTAAG & ATTAGCTTGTTGTTCCCGCC & NM_022I85.2 \\
GAPDH & CGCTAACATCAAATGGGGTG & TTGCTGACAATCTTGAGGGAG & NM_017008.4R \\
microRNA-I26 & TCGTACCGTGAGTAATAATGCG & CTCAACTGGTGTCGTGGAGTC & MIMAT0000832 \\
U6 & CCTGCTTCGGCAGCACAT & AACGCTTCACGAATTTGCGT & K00784.I \\
\hline
\end{tabular}

SPRED1 (1:1,000; Abcam), mouse anti-VEGF (1:500; Abcam), and rabbit anti-glyceraldehyde 3-phosphate dehydrogenase (1:1,000; Abcam). Next, anti-rabbit IgG (1:10,000; Aspen, Inc.) and anti-mouse $\operatorname{IgG}(1: 10,000$; Aspen, Inc.) were used as second antibodies at 1:2,000 for $30 \mathrm{~min}$. Quantification of the protein bands were performed with AlphaEaseFC (Alpha Innotech, San Leandro, CA) software. Sample analyses were performed on day 9.

\section{Statistical analysis}

The data were presented as the mean \pm standard deviation and analyzed with SPSS 18.0 (SPSS Inc., Chicago, IL, USA). Numerical variables with variance homogeneity were performed using two-sample $t$-test. Pearson correlation analysis was employed to assess the correlation between normally distributed data. A probability value $(P)$ of less than 0.05 was considered to be a statistically significant difference.

\section{Results}

\section{The acceleration of wound healing in NPWT group}

Wound healing was significantly accelerated by NPWT. Photographs of wound healing progression from each group in diabetic wound model were shown in Figure 1A.
Obviously, diabetic wounds treated with NPWT showed significantly healing rates at all time points compared with control groups $(P<0.05$, Figure 1B). More importantly, as exhibited in Figure 2, when compared with control group, NPWT group shown abundant blood vessel distribution, increased collagen fibers aggregation and less inflammatory cells on days $3,5,7$, and 9 via using (H\&E) staining.

\section{Increased angiogenesis in NPWT group}

$\alpha$-SMA-positive cells per square millimeter were used to evaluate arteriolar density, and the results are summarized in Figure 3. Arteriolar density was significantly increased in the NPWT group compared with the control group at each time point $(3,5,7,9$ days; $P<0.05$; Figure 5A). Using CD31 staining to assess capillary density, we observed abundant CD31-positive cells per square millimeter in the NPWT group but few cells in the control group on days3, 5, 7, and $9(P<0.05$; Figure 4, Figure 5B).

\section{NPWT increases miR-I 26 expression in both tissue and plasma obtained from a diabetic rat wound model}

We examined miR-126 expression from wound tissue and plasma obtained from the diabetic rat wound model treated
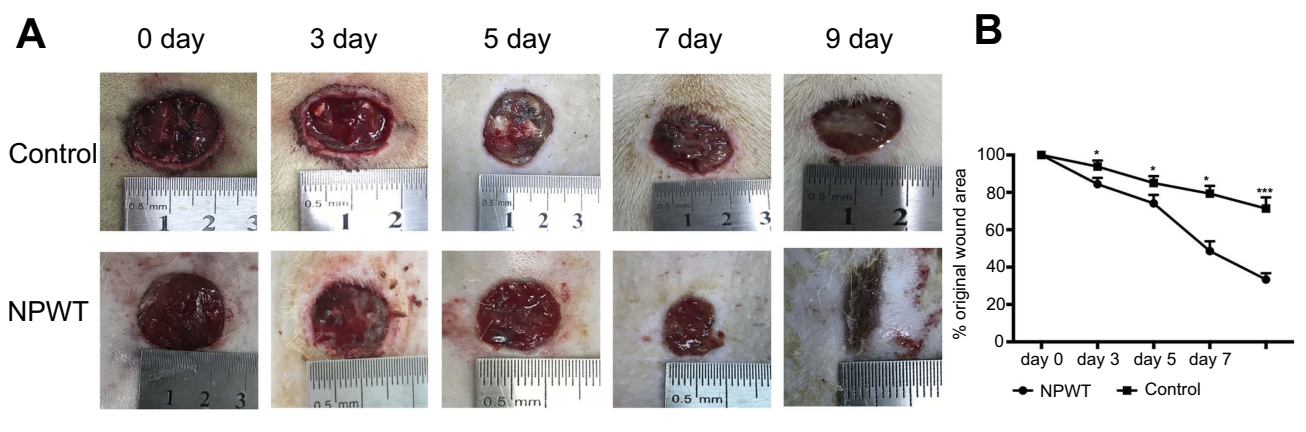

Figure I (A) Representative wound images in NPWT group and control group at day 0, 3, 5, 7, 9. (B) Percent wound closure. Data is given as the mean \pm SD, $* P<0.05$, $* * P<0.01$.

Abbreviations: NPWT, negative pressure wound therapy group; Control, control group. 


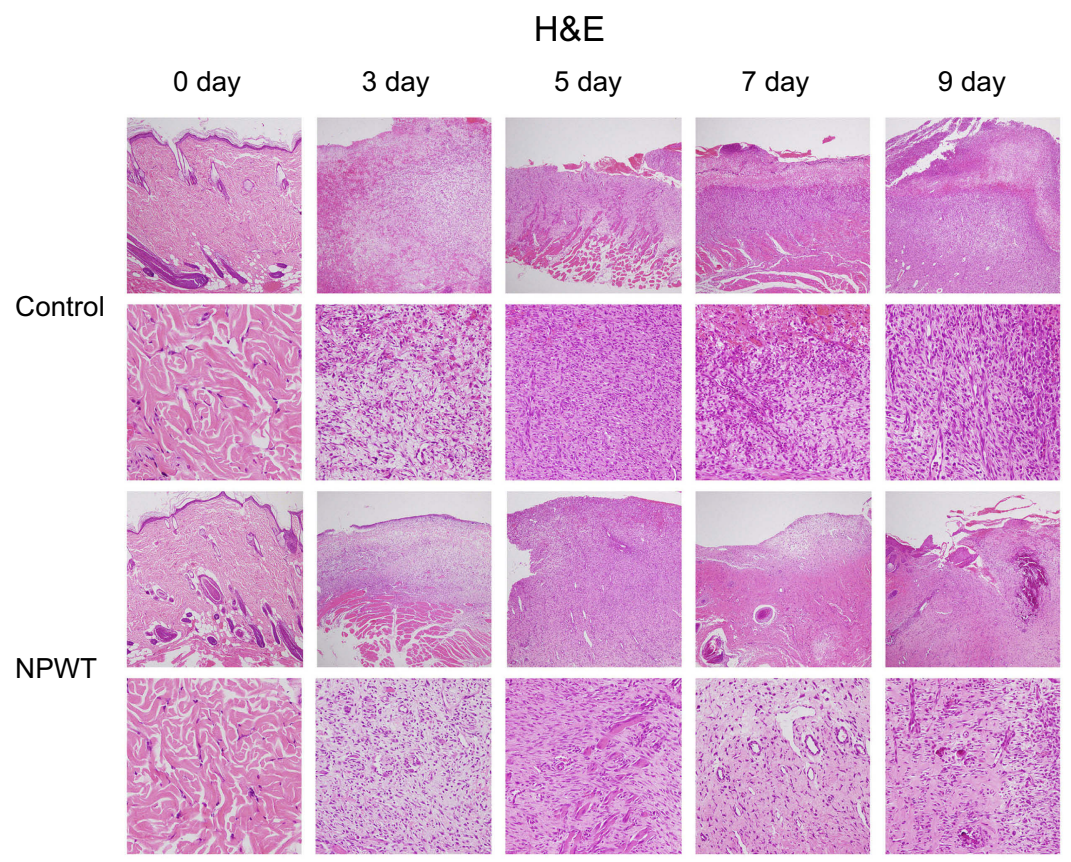

Figure $2 \mathrm{H \& E}$ staining (40xmagnification or 200xmagnification) in NPWT group and control group at day 0, 3, 5, 7, 9. Abbreviations: H\&E, hematoxylin and eosin stain; NPWT, negative pressure wound therapy group; Control, control group.

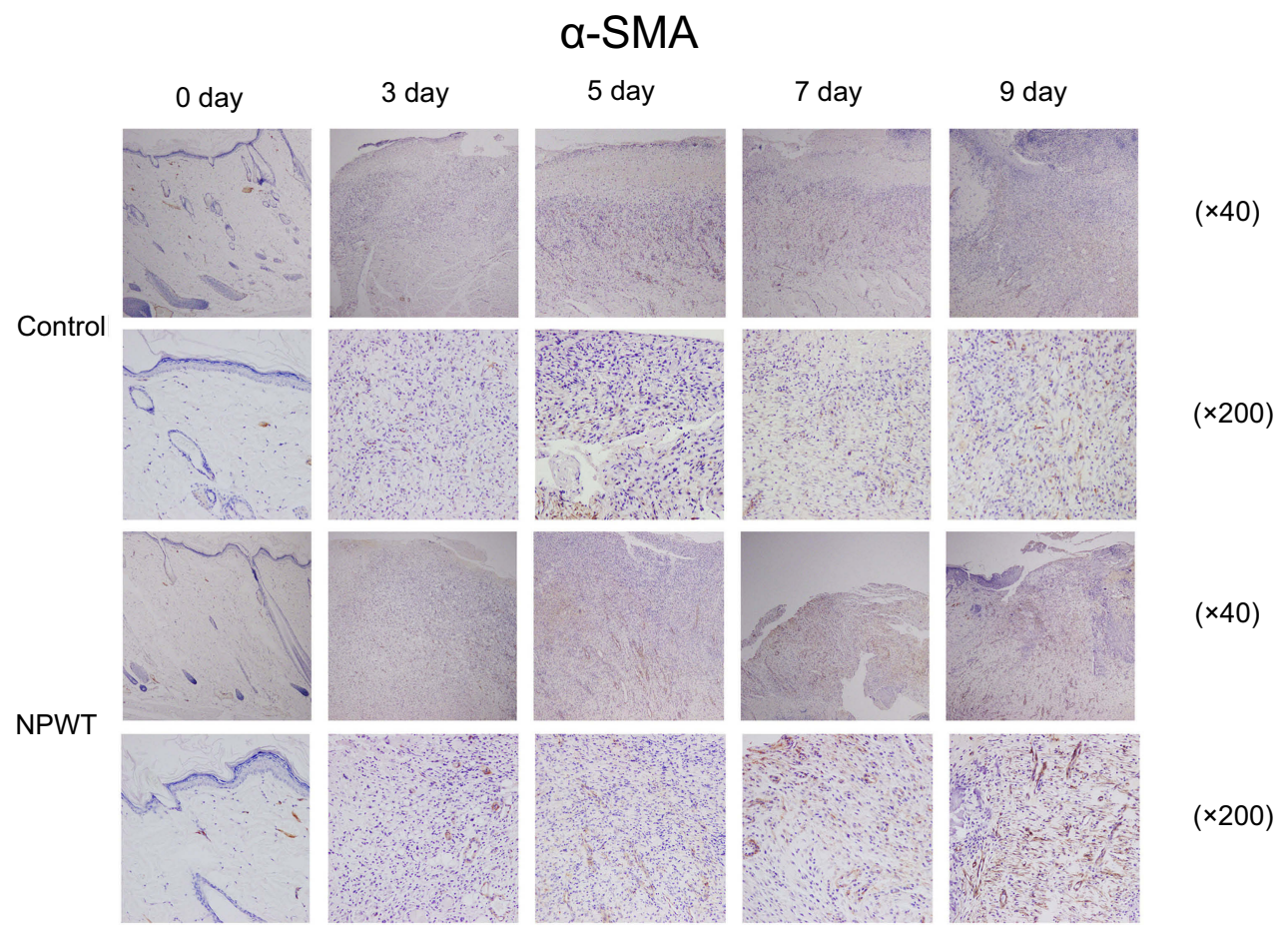

Figure 3 Immunohistochemistry (40xmagnification or 200xmagnification) for the expression of $\alpha$-SMA representing freshly formed blood vessels at day 0,3 , 5, 7, and 9 postwounding. Abbreviations: $\alpha$-SMA, $\alpha$ smooth muscle actin; NPWT, negative pressure wound therapy group; Control, control group.

with NPWT and conventional dressing on days $0,3,5,7, \quad$ group at every time point $(3,5,7$, and 9 days; $P<0.05$; and 9. The results were presented in Figure 2A and B. Figure 6A). In addition, miR-126 expression in the plasma Tissue miR-126 expression levels were significantly samples from the NPWT group also exhibited significantly increased in the NPWT group compared with the control increased levels compared with the control group on days 


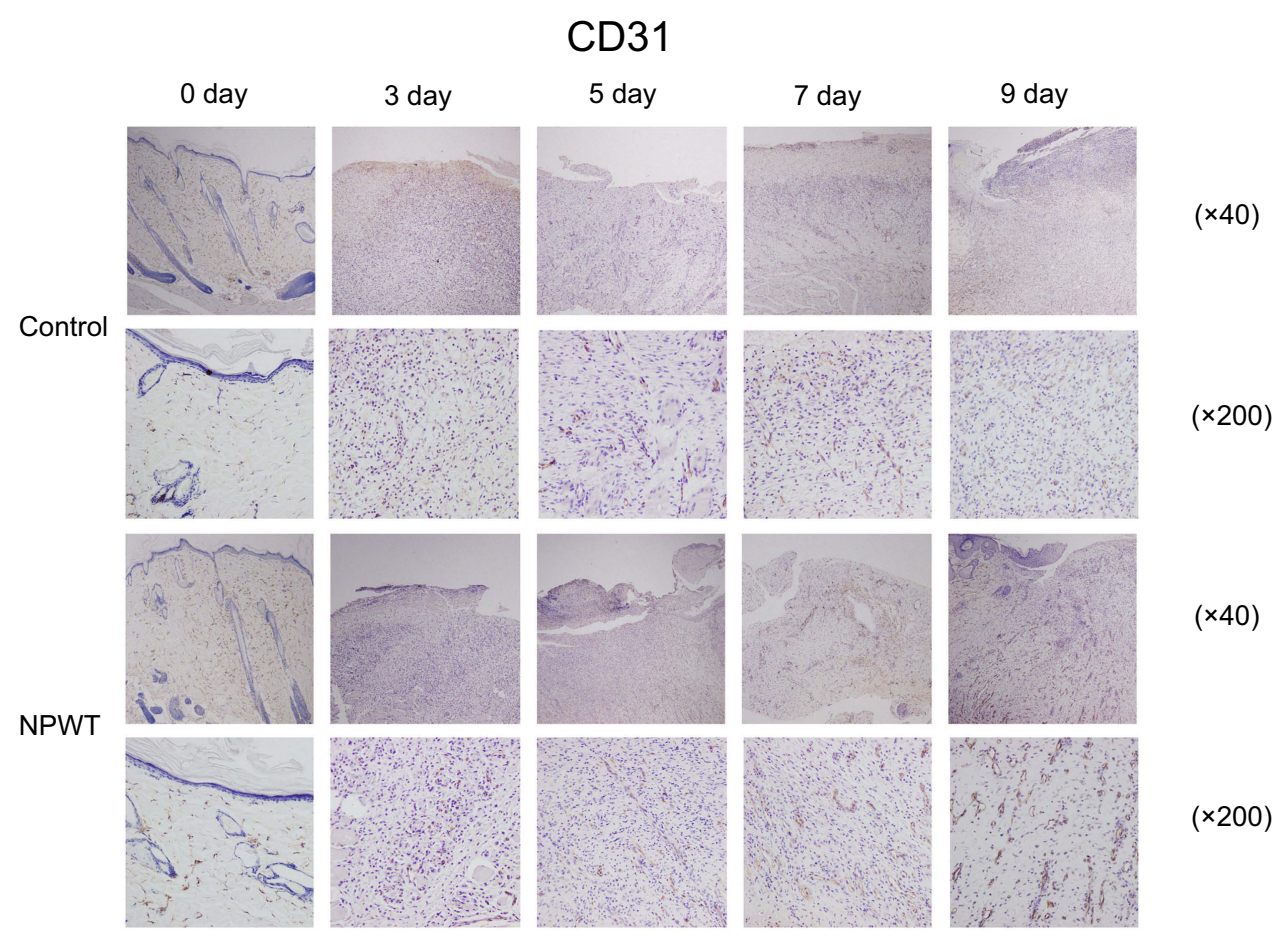

Figure 4 Immunohistochemistry (40xmagnification or 200xmagnification) for the expression of CD3। representing freshly formed blood vessels at day $0,3,5,7$, and 9 postwounding.

Abbreviations: CD3I, Platelet endothelial cell adhesion molecule-I; NPWT, negative pressure wound therapy group; Control, control group.

A

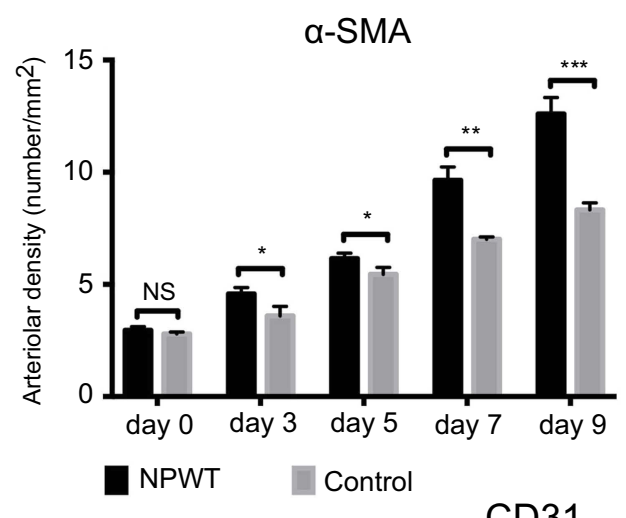

B

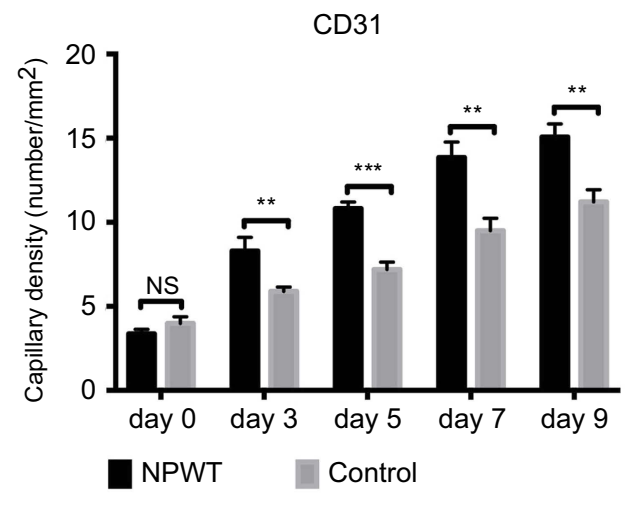

Figure 5 (A and B) Quantification of arteriolar density and capillary density in wound tissue of control and NPWT-treated diabetic rats on days 0, 3, 5, 7, and 9 postwounding. Data are expressed as the mean \pm SD, NS $>0.05$, $* P<0.05, * * * P<0.001$.

Abbreviations: $\alpha$-SMA, $\alpha$ smooth muscle actin; CD3I, Platelet endothelial cell adhesion molecule-I; NPWT, negative pressure wound therapy group; Control, control group.

$3,5,7$, and $9(P<0.05$; Figure 6B). Furthermore, tissue and plasma miR-126 levels exhibited a significant increase compared with baseline level ( 0 day) in the NPWT group. Moreover, in the NPWT group, a very significant positive correlation was noted between miR-126 detected in wound tissue and its expression in plasma via linear regression analysis $(\mathrm{r}=0.871 ; P<0.01$; Figure $6 \mathrm{C})$.
miR-I 26 modulates VEGF receptor pathway activity in the diabetic rat wound model treated with NPWT

In our study, VEGF mRNA and protein levels were significantly increased on day 9 in the NPWT group (Figure 7A-C). Previous studies demonstrated that miRNA-126 facilitates 
A

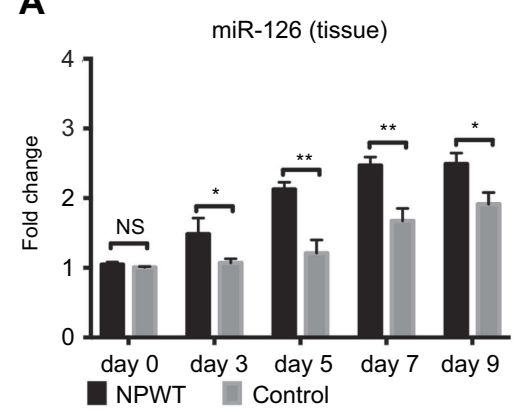

B

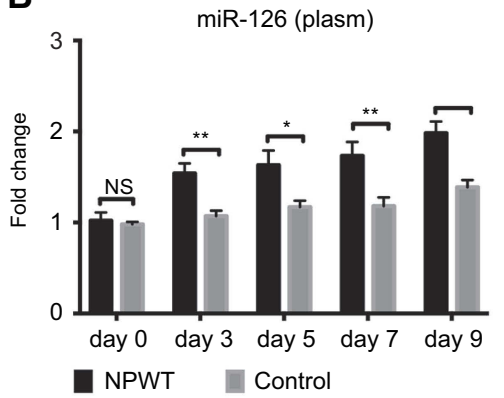

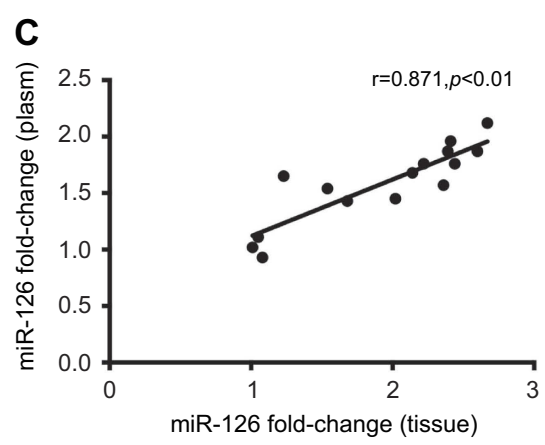

Figure 6 (A and B) Effects of NPWT on miRNA-I 26 expression levels in wound tissue and plasma compared with the control group as assessed by qRT-PCR on days $0,3,5$, 7 and 9 postwounding. U6 was used as an endogenous control. (C) In the NPWT group, miRNA-I 26 expression levels in the plasma was associated with levels in wound tissue $(r=0.87 \mathrm{I}, P<0.0 \mathrm{I})$. NS $>0.05, * P<0.05$, $* * P<0.01$.

Abbreviations: NPWT, negative pressure wound therapy group; Control, control group.

A

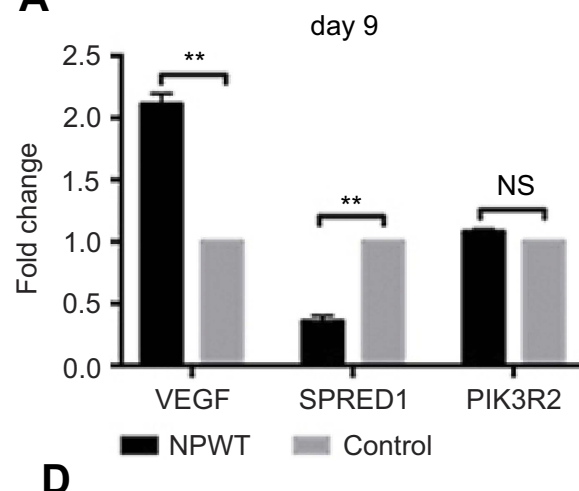

D

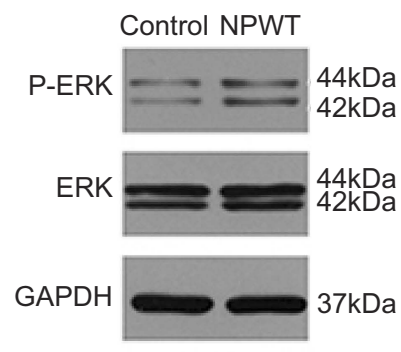

B

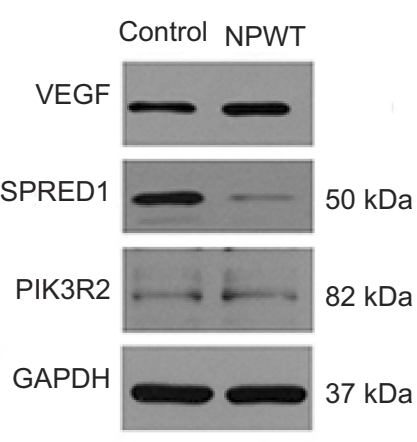

$\mathbf{E}$

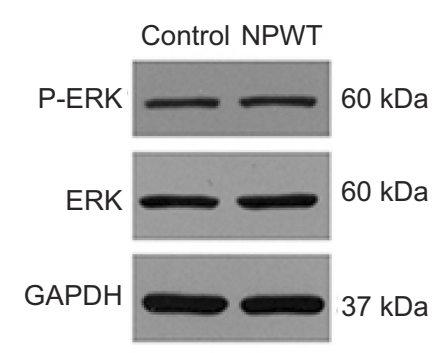

C

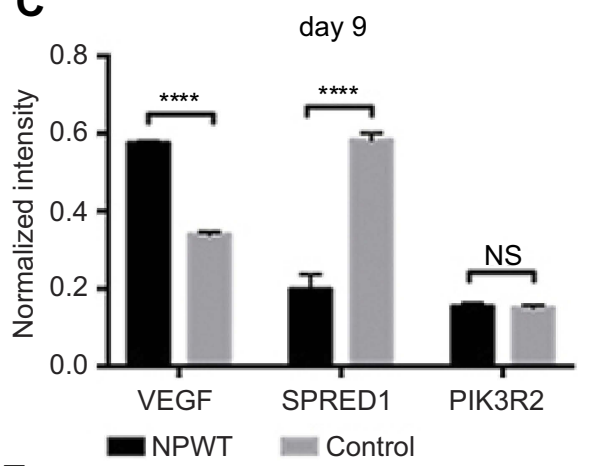

$\mathbf{F}$

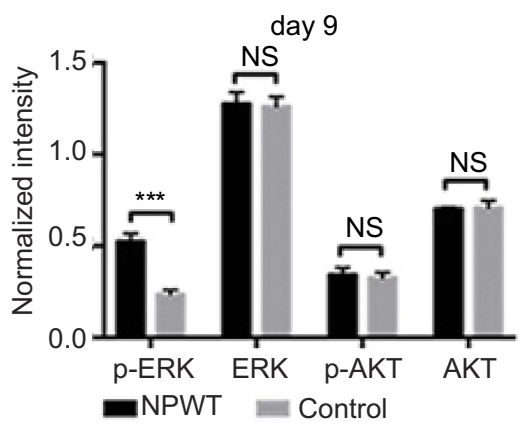

Figure 7 (A) qRT-PCR analysis of VEGF, SPREDI and PIK3R2 gene expression in the NPWT and control groups on day 9. GAPDH was used as an endogenous control. (B) Western blotting of VEGF, SPREDI and PIK3R2 in granulation tissue in the control and NPWT group on day 9. (C) Quantification of VEGF, SPREDI and PIK3R2 protein expression in the NPWT versus control group on day 9. (D and E) Western blotting of p-ERK, ERK, p-AKT and AKT in wound healing tissue of the control and NPWT group on day 9. (F) Quantification of protein expression levels of p-ERK, ERK, p-AKT and AKT in the NPWT group versus control group on day 9. Proteins were normalized to GAPDH on day 9 , and values are reported as relative change compared with control. NS $>0.05, * * P<0.01, * * * P<0.001, * * * * P<0.0001$.

Abbreviations: VEGF, vascular endothelial growth factor; SPREDI, Sprouty-related EVHI domain containing protein I; PIK3R2, phosphoinositol-3 kinase regulatory subunit 2; GAPDH, glyceraldehyde-3-phosphate dehydrogenase; ERK, extracellular regulated protein kinases; p-ERK, phosphorylate extracellular regulated protein kinases; AKT, protein kinase B; P-AKT,phosphorylateprotein kinase B; NPWT, negative pressure wound therapy group; Control, control group.

angiogenesis by inhibiting SPRED1 and PIK3R2, two negative regulators of the VEGF receptor pathway. To investigate potential mechanism underlying the influence of NPWT on diabetic rat wound models, we detected mRNA levels of miRNA-126 target genes and corresponding protein levels using qRT-PCR and Western blotting, respectively, on day 9
(Figure 7). The NPWT group exhibited significantly reduced SPRED1 mRNA expression levels. The result was consistent with a previous study that demonstrated that increased miR126 levels cause SPRED1 downregulation (Figure 7A). ${ }^{17}$ SPRED1 protein levels correlated with the mRNA changes (Figure $7 \mathrm{~B}-\mathrm{C}$ ). When compared with the control group on 
9 days, treatment with NPWT significantly increased pERK protein levels, which is negatively regulated by SPRED1 (Figure 7D and F).

However, we found that the mRNA and protein levels of PIK3R2, which is validated target genes of miR-126, were not significantly different between the NPWT group and control group on 9 days (Figure 7A-C). NPWT did not significantly increased pAKT/AKT expression compared with the conventional dressing group (Figure 7E-F).

\section{Association of circulating miR-I 26 with capillary density and arteriolar density in the diabetic rat wound model treated with NPWT}

Vessel density increased upon upregulation circulating miR-126 upregulated at the four time points in the NPWT group. A positive correlation was observed between circulating miR-126 levels and arteriolar density in the diabetic rat wound model treated with NPWT ( $\mathrm{r}=0.827, P<0.01$, Figure $8 \mathrm{~A}$ ). The same observation was noted concerning capillary density and circulating miR126 with a correlation coefficient of $0.895 \quad(P<0.01$, Figure 8B).

\section{Discussion}

At present, mounting evidence suggests that the use of NPWT is beneficial for nonhealing diabetic wounds by promoting angiogenesis. ${ }^{24,25}$ However, the precise mechanism of NPWT-induced angiogenesis is less obvious. In our study, we demonstrated that miR-126 may participate in NPWT-induced angiogenesis in diabetic wounds by regulating the VEGF receptor pathway.
Furthermore, we identified a significant correlation between circulating miR-126 and angiogenesis, laying the foundation for further clinical application.

The intractable wound of diabetic patients is one leading cause of diabetes-associated morbidity and mortality, which presents an enormous challenge to all surgeons. ${ }^{26}$ As described above, angiogenesis is an essential process of wound repair given that vessels can provide nutrition and oxygen for cells at the wound site and remove waste products. However, the pathogenic mechanisms of nonhealing diabetic wounds and other micro/macrovascular diabetic complications largely involve insufficient angiogenesis; therefore, the acceleration of wound healing remains precarious.

NPWT has been widely used in clinical practice to increase wound healing for decades. The therapy includes the controlled application of sub-atmospheric pressure to the local wound environment using a sealed wound dressing connected to a vacuum pump. Numerous studies have revealed that the facilitation of angiogenesis surrounding the wound bed is one of the most significant beneficial factors of NPWT. ${ }^{7}$ NPWT-induced angiogenesis occurs via VEGF pathway activation in the wound area, where VEGF overexpression has been detected. ${ }^{27,28}$ As one of the most significant growth factors, VEGF increases angiogenesis in diabetic wounds. ${ }^{29}$ Our results were consistent with the previous studies and provided evidence that NPWT promotes VEGF-induced angiogenesis. In addition, our previous studies demonstrated that NPWT increased capillary quality via promoting structural integrity and functional stabilization at various stages of wound healing. ${ }^{30}$ However, exogenous application of VEGF as a proangiogenic factor did not exhibit a reliable clinical
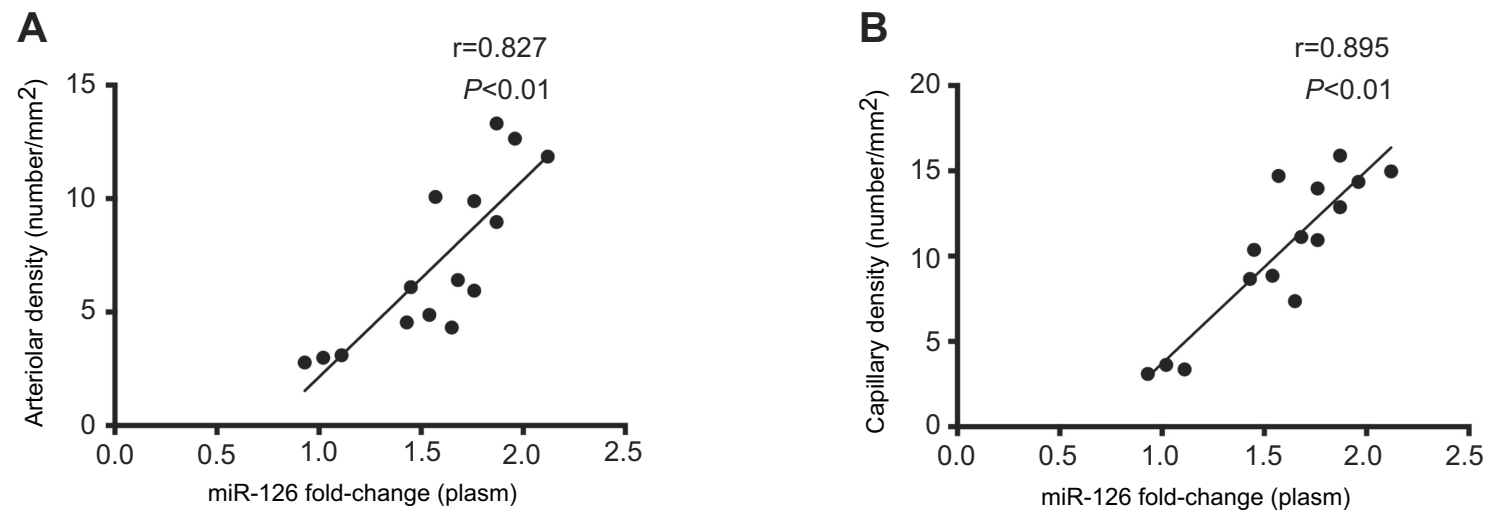

Figure 8 (A and B) In the NPWT group, the correlation between circulating miR-I 26 expression and quantitative indexes of angiogenesis. Significantly positive association was detected between circulating miR-I 26 and arteriolar density $(\mathbf{A})(r=0.827, P<0.01)$ or capillary density $(\mathbf{B})(r=0.895, P<0.01)$. Statistical analysis was performed using the Pearson correlation coefficient. 
effect, which may lead to sustained leakage of vascularization and disorganized formation of blood vessels. This treatment was abandoned after phase II clinical trials. ${ }^{31}$ Therefore, it is worth exploring the possible mechanism by which NPWT promotes VEGF expression and vascular maturation.

Given that miRNAs have more than one target, they are referred to as powerful gene expression regulators of an entire functional network. To date, the vast majority of miRNAs are key mediators in the phases of wound repair. $^{13,32}$ Here, we focus on the roles of miR-126 in wound healing using NPWT. miR-126, which is located in an intron of $E g f l 7$, is abundantly enriched in endothelial cells. ${ }^{33}$ Previous studies have demonstrated that angiogenesis does not occur in zebrafish and mouse embryogenesis when miR-126 is knocked out. ${ }^{16}$ In addition, emerging evidence suggests that miR-126 plays an important role in the stabilization and maturation of neovascularization by influencing angiopoietin-1 signaling and p21-activated kinase 1 gene expression. ${ }^{34,35}$ Studies have also found that miR-126 facilitates angiogenesis after injury via regulating SPRED1 and PIK3R2 expression (also known as p85 $\beta$ ), which are two negative regulators of the VEGF pathway. ${ }^{16,33}$ The miR-126SPRED1/PIK3R2-VEGF axis has been assessed as a dominant regulator in the process of angiogenesis. ${ }^{18,36}$ SPRED1 is a member of the Sprouty and Sprouty-related protein family and negatively regulates MAP kinase pathway activation. ${ }^{33}$ Another target of miR-126, PIK3R2, inhibits activation of PI3 kinase, which is critical in the AKT pathway. ${ }^{37}$ The PI3 kinase pathway and the MAP kinase pathway, which can be evaluated by quantifying pAKT and pERK, respectively, are activated in response to miR-126. Numerous studies have revealed that miR126 performs an insignificant role in the different phases of wound repair. miR-126 regulates leukocyte adherence to endothelial cells and the expression of vascular cell adhesion molecule-1 (VCAM)-1 in the inflammatory phase. ${ }^{38}$ In the proliferation phase, miR-126 is important for endothelial cell proliferation, migration and angiogenesis. $^{33}$ More importantly, miR-126 was downregulated in diabetic patients and animal models, which hindered endothelial progenitor cell function. ${ }^{39}$ What's more, the lower expression was observed in patients with diabetic micro/macrovascular complications, which indicates impaired angiogenesis in these individuals. ${ }^{18,19}$ However, upregulation of miR-126 expression via various mechanisms partly restored wound repair ability and angiogenesis. ${ }^{15,21,40}$ These data demonstrate an indispensable role of miR-126 in repair and regeneration of diabetic tissue healing. Given that the key role of miR126 in angiogenesis and vessel maturation of injury repair occurs by regulating multiple signaling pathways, miR-126 overexpression contributes to diabetic wound healing. Our study demonstrated that miR-126 tissue levels were upregulated in the NPWT group on days 3, 5, 7, and 9. In addition, reduced SPRED1 expression was noted on day 9 compared with the conventional dressing group. In the NPWT group, the reduction of SPRED1 expression, which facilitates the MAP kinase pathway, was associated with overexpression of pERK, which is downstream of the pathway. However, the expression of another target of miR-126, PIK3R2, and its downstream pathway did not exhibit any differences between the NPWT group and conventional treatment group. Thus, our research contributes to evidence to the hypothesis that miR-126 plays a regulatory role in VEGF-induced angiogenesis of diabetic wounds treated with NPWT by affecting the expression of SPRED1 and the MAP kinase pathway. Our study also suggests that NPWT may foster neovascular stabilization and maturation by increasing miR-126 expression.

We previously demonstrated that NPWT promotes VEGFinduced angiogenesis of wounds, which was accompanied by the overexpression of miR-126 in wound tissue as demonstrated in this study. Furthermore, miRNAs have been found in stable forms in different body fluids, such as plasma and serum, where their changes appear to represent disease states or the response to certain therapies. ${ }^{41}$ Although the source of circulating miRNA is still debated, emerging evidence suggests that these circulating miRNAs are from tissues in the context of pathological stress, including wound tissue. ${ }^{41}$ Several underlying mechanisms are involved in this phenomenon, including leakage from damaged cells, microvesicles secretion, or microvesicles-free mechanisms. ${ }^{42}$ Our research revealed that miR-126 expression was roughly similar between plasma and wound tissue in the NPWT group. Our data also suggest that circulating miR-126 likely originated from wound tissue. Given its specific expression, miR-126 holds great potential as a biomarker for diagnosis, prognosis, and monitoring of treatment effects. Currently, the methods available to assess diabetic wound angiogenesis are typically rely on significant clinical experience. Thus, we explored the expression of circulating miR-126 and its clinical relevance in NPWT-induced angiogenesis. Our research demonstrated that circulating miR-126 expression was significantly increased in 
the NPWT group compared with the control group at every time point. More importantly, its expression was markedly associated with two quantitative indexes of angiogenesis in a diabetic wound model treated with NPWT. Given its high level and long duration of expression, we hypothesize that circulating miR-126 can be a more precise and innovative tool for monitoring nonhealing diabetic wound angiogenesis under the conditions of NPWT instead frequent changes of dressings, causing an unnecessary economic burden and pain. Therefore, measurement of miR-126 seems to be of value for distinguishing between successful and failed angiogenesis of nonhealing complex wounds of diabetic patients during NPWT in the future.

In conclusion, our study revealed significant increases in miR-126, which regulates VEGF-induced angiogenesis in diabetic wounds treated with NPWT. Determination of circulating miR-126 concentrations might be valuable for predicting the level of angiogenesis in diabetic wounds treated with NPWT. However, our research was based on animal models that insufficiently approximate human diabetic wound healing, especially chronic wounds. Therefore, further studies are essential to detect standardized and detailed expression levels of miR-126 in clinical samples and the exact mechanism controlling SPRED1 expression. We may also need to further investigate the combinatorial therapeutic effect between modulating the miR-126 expression levels and NPWT on nonhealing diabetic wounds in the future.

\section{Acknowledgments}

The authors would like to acknowledge the Wuhan VSD Medical Science \& Technology, Co., Ltd. (Wuhan, China) for supplying the vacuum material. This work was supported by Grants from the Independent Research Project of Wuhan University (Grant no. 2042017kf0079).

\section{Disclosure}

Dr Zonghuan li, Dr Zheng Wang, Dr Weidong Xiao, Dr Aixi Yu report grants from the Independent Research Project of Wuhan University (Grant no. 2042017kf0079), during the conduct of the study. The authors report no other conflicts of interest in this work.

\section{References}

1. Ali SR, Ozdemir BA, Hinchliffe RJ. Critical appraisal of the quality of evidence addressing the diagnosis, prognosis, and management of peripheral artery disease in patients with diabetic foot ulceration. Eur J Vasc Endovasc Surg. 2018;56(3):401-408. doi:10.1016/j.ejvs.2018.05.009

2. Brem H, Tomic-Canic M. Cellular and molecular basis of wound healing in diabetes. J Clin Invest. 2007;117(5):1219-1222. doi:10.1172/JCI32169
3. Eming SA, Martin P, Tomic-Canic M. Wound repair and regeneration: mechanisms, signaling, and translation. Sci Transl Med. 2014;6 (265):265sr266-265sr266. doi:10.1126/scitranslmed.3009337

4. Okonkwo U, DiPietro L. Diabetes and wound angiogenesis. Int J Mol Sci. 2017;18(7):1419. doi:10.3390/ijms18071419

5. Erba P, Ogawa R, Ackermann M, et al. Angiogenesis in wounds treated by microdeformational wound therapy. Ann Surg. 2011;253 (2):402-409. doi:10.1097/SLA.0b013e31820563a8

6. Argenta LC, Morykwas MJ. Vacuum-assisted closure: a new method for wound control and treatment: clinical experience. Ann Plast Surg. 1997;38(6):563-576; discussion 577.

7. Jacobs S, Simhaee DA, Marsano A, Fomovsky GM, Niedt G, Wu JK. Efficacy and mechanisms of vacuum-assisted closure (VAC) therapy in promoting wound healing: a rodent model. JPRAS. 2009;62 (10):1331-1338. doi:10.1016/j.bjps.2008.03.024

8. Nissen NN, Polverini PJ, Koch AE, Volin MV, Gamelli RL, DiPietro LA. Vascular endothelial growth factor mediates angiogenic activity during the proliferative phase of wound healing. Am J Pathol. 1998;152(6): 1445-1452.

9. Yan X, Chen B, Lin Y, et al. Acceleration of diabetic wound healing by collagen-binding vascular endothelial growth factor in diabetic rat model. Diabetes Res Clin Pract. 2010;90(1):66-72. doi:10.1016/j. diabres.2010.07.001

10. Li Z, Yu A. Complications of negative pressure wound therapy: a mini review. Wound Repair Regen. 2014;22(4):457-461. doi:10.1111/ wrr.12190

11. Bartel DP. MicroRNAs: genomics, biogenesis, mechanism, and function. Cell. 2004;116(2):281-297.

12. Ambros V. The functions of animal microRNAs. Nature. 2004;431 (7006):350-355. doi:10.1038/nature02871

13. Roy S, Sen CK. miRNA in wound inflammation and angiogenesis. Microcirculation. 2012;19(3):224-232. doi:10.1111/j.15498719.2011.00156.x

14. Nicoli S, Standley C, Walker P, Hurlstone A, Fogarty KE, Lawson ND. MicroRNA-mediated integration of haemodynamics and Vegf signalling during angiogenesis. Nature. 2010;464(7292):1196-1200. doi:10.1038/nature08889

15. Hu J, Zeng L, Huang J, Wang G, Lu H. miR-126 promotes angiogenesis and attenuates inflammation after contusion spinal cord injury in rats. Brain Res. 2015;1608:191-202. doi:10.1016/j.brainres.2015.02.036

16. Fish JE, Santoro MM, Morton SU, et al. miR-126 regulates angiogenic signaling and vascular integrity. Dev Cell. 2008;15(2):272-284. doi:10.1016/j.devcel.2008.07.008

17. Wang S, Aurora AB, Johnson BA, et al. The endothelial-specific microRNA miR-126 governs vascular integrity and angiogenesis. Dev Cell. 2008;15(2):261-271. doi:10.1016/j.devcel.2008.07.002

18. Rawal S, Munasinghe PE, Shindikar A, et al. Down-regulation of proangiogenic microRNA-126 and microRNA-132 are early modulators of diabetic cardiac microangiopathy. Cardiovasc Res. 2017;113 (1):90-101. doi:10.1093/cvr/cvw235

19. Barutta F, Bruno G, Matullo G, et al. MicroRNA-126 and micro-/ macrovascular complications of type 1 diabetes in the EURODIAB prospective complications study. Acta Diabetol. 2017;54(2):133-139. doi:10.1007/s00592-016-0915-4

20. Rezk NA, Sabbah NA, Saad MS. Role of MicroRNA 126 in screening, diagnosis, and prognosis of diabetic patients in Egypt. IUBMB Life. 2016;68(6):452-458. doi:10.1002/iub.1502

21. Zhang J, Sun XJ, Chen J, et al. Increasing the miR-126 expression in the peripheral blood of patients with diabetic foot ulcers treated with maggot debridement therapy. J Diabetes Complications. 2017;31 (1):241-244. doi:10.1016/j.jdiacomp.2016.07.026

22. Jansen F, Yang X, Hoelscher M, et al. Endothelial microparticlemediated transfer of MicroRNA-126 promotes vascular endothelial cell repair via SPRED1 and is abrogated in glucose-damaged endothelial microparticles. Circulation. 2013;128(18):2026-2038. doi:10.1161/CIRCULATIONAHA.113.001720 
23. Chamorro-Jorganes A, Araldi E, Suarez Y. MicroRNAs as pharmacological targets in endothelial cell function and dysfunction. Pharmacol Res. 2013;75:15-27. doi:10.1016/j.phrs.2013.04.002

24. Dumville JC, Hinchliffe RJ, Cullum N, et al. Negative pressure wound therapy for treating foot wounds in people with diabetes mellitus. Cochrane Database Syst Rev. 2013;10(10):Cd010318.

25. Dumville JC, Land L, Evans D, Peinemann F. Negative pressure wound therapy for treating leg ulcers. Cochrane Database Syst Rev. 2015;14;(7):CD011354. doi:10.1002/14651858.CD011354.

26. Nelson EA, O'Meara S, Craig D, et al. A series of systematic reviews to inform a decision analysis for sampling and treating infected diabetic foot ulcers. Health Technol Assess. 2006;10(12):iii-iv, ix-x, $1-221$.

27. Li X, Liu J, Liu Y, et al. Negative pressure wound therapy accelerates rats diabetic wound by promoting agenesis. Int $J$ Clin Exp Med. 2015;8(3):3506-3513.

28. Xia CY, Yu AX, Qi B, Zhou M, Li ZH, Wang WY. Analysis of blood flow and local expression of angiogenesisassociated growth factors in infected wounds treated with negative pressure wound therapy. Mol Med Rep. 2014;9(5):1749-1754. doi:10.3892/mmr.2014.1997

29. Fitch MJ, Campagnolo L, Kuhnert F, Stuhlmann H. Egfl7, a novel epidermal growth factor-domain gene expressed in endothelial cells. Dev Dyn. 2004;230(2):316-324. doi:10.1002/dvdy.20063

30. Ma Z, Shou K, Li Z, Jian C, Qi B, Yu A. Negative pressure wound therapy promotes vessel destabilization and maturation at various stages of wound healing and thus influences wound prognosis. Exp Ther Med. 2016;11(4):1307-1317. doi:10.3892/etm.2016. 3083

31. Barrientos S, Brem H, Stojadinovic O, Tomic-Canic M. Clinical application of growth factors and cytokines in wound healing. Wound Repair Regen. 2014;22(5):569-578. doi:10.1111/wrr.12205

32. Herter EK, Xu Landen N. Non-coding RNAs: new players in skin wound healing. Adv Wound Care. 2017;6(3):93-107. doi:10.1089/ wound.2016.0711

33. van Solingen C, Seghers L, Bijkerk R, et al. Antagomir-mediated silencing of endothelial cell specific microRNA-126 impairs ischemia-induced angiogenesis. J Cell Mol Med. 2009;13(8a):1577-1585. doi:10.1111/j.1582-4934.2008.00613.x
34. Sessa R, Seano G, Di Blasio L, et al. The miR-126 regulates angiopoietin-1 signaling and vessel maturation by targeting p85beta. Biochim Biophys Acta. 2012;1823(10):1925-1935. doi:10.1016/j. bbamcr.2012.07.011

35. Zou J, Li WQ, Li Q, et al. Two functional microRNA-126s repress a novel target gene p21-activated kinase 1 to regulate vascular integrity in zebrafish. Circ Res. 2011;108(2):201-209. doi:10.1161/ CIRCRESAHA.110.225045

36. Park CW, Kim HW, Lim JH, et al. Vascular endothelial growth factor inhibition by dRK6 causes endothelial apoptosis, fibrosis, and inflammation in the heart via the Akt/eNOS axis in $\mathrm{db} / \mathrm{db}$ mice. Diabetes. 2009;58(11):2666-2676. doi:10.2337/db09-0136

37. Ueki K, Fruman DA, Yballe CM, et al. Positive and negative roles of p85alpha and p85beta regulatory subunits of phosphoinositide 3kinase in insulin signaling. $J$ Biol Chem. 2017;292(13):5608. doi:10.1074/jbc.A117.305602

38. Ortega FJ, Mercader JM, Moreno-Navarrete JM, et al. Profiling of circulating microRNAs reveals common microRNAs linked to type 2 diabetes that change with insulin sensitization. Diabetes Care. 2014;37(5):1375-1383. doi:10.2337/dc13-1847

39. Meng S, Cao JT, Zhang B, Zhou Q, Shen CX, Wang CQ. Downregulation of microRNA-126 in endothelial progenitor cells from diabetes patients, impairs their functional properties, via target gene Spred-1. J Mol Cell Cardiol. 2012;53(1):64-72. doi:10.1016/j. yjmcc.2012.04.003

40. Tsumaru S, Masumoto H, Minakata K, et al. Therapeutic angiogenesis by local sustained release of microRNA-126 using poly lacticco-glycolic acid nanoparticles in murine hindlimb ischemia. $J$ Vasc Surg. 2018;68(4):1209-1215.

41. Laterza OF, Lim L, Garrett-Engele PW, et al. Plasma MicroRNAs as sensitive and specific biomarkers of tissue injury. Clin Chem. 2009;55(11):1977-1983. doi:10.1373/clinchem.2009.131797

42. Chen X, Liang H, Zhang J, Zen K, Zhang CY. Secreted microRNAs: a new form of intercellular communication. Trends Cell Biol. 2012;22 (3):125-132. doi:10.1016/j.tcb.2011.12.001 


\section{Supplementary materials}
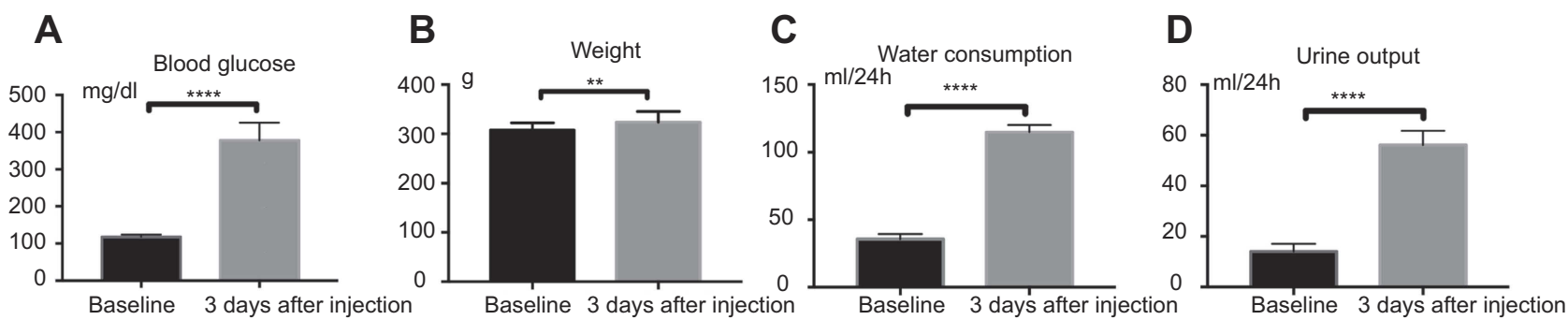

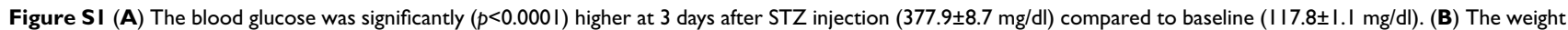
at 3 days post injection was $323.8 \pm 3.9 \mathrm{~g}$, which significantly $(p<0.01)$ increased compared to the body weight on baseline $(308.4 \pm 2.6 \mathrm{~g})$. (C) The water consumption significantly $(p<0.000 \mathrm{I})$ raised after injection $(1 \mathrm{I} 4.8 \pm \mathrm{I} .0 \mathrm{ml} / 24 \mathrm{~h})$ compared to baseline $(35.5 \pm 0.7 \mathrm{ml} / 24 \mathrm{~h})$ (D) The urine output was significantly $(p<0.000 \mathrm{I})$ elevated at 3 days after injection $(56.1 \pm 1.0 \mathrm{ml} / 24 \mathrm{~h})$ in comparison to baseline $(14.1 \pm 0.6 \mathrm{ml} / 24 \mathrm{~h}) \square * * P<0.01, * * * * p<0.0001$.

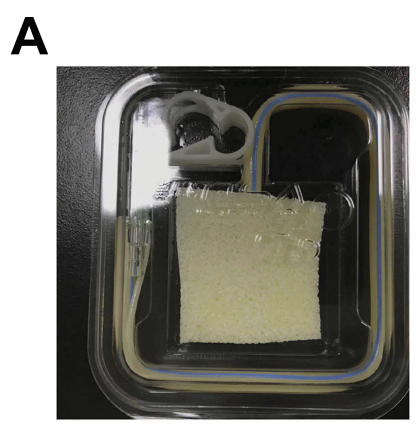

B

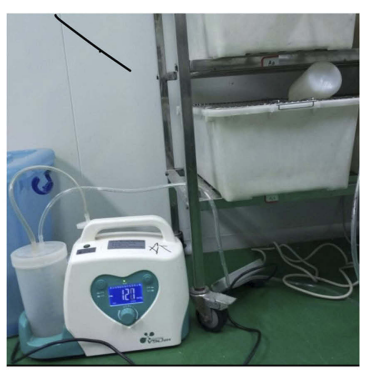

Figure S2 (A) The wound was covered with a medical foam (VSD Medical Technology Inc., Wuhan, China). (B) Constant 125 mmHg suction was produced by a Vacuumassisted Closure Device (VSD Medical Technology Inc., Wuhan, China).

\section{Publish your work in this journal}

Diabetes, Metabolic Syndrome and Obesity: Targets and Therapy is an international, peer-reviewed open-access journal committed to the rapid publication of the latest laboratory and clinical findings in the fields of diabetes, metabolic syndrome and obesity research. Original research, review, case reports, hypothesis formation, expert opinion and commentaries are all considered for publication. The manuscript management system is completely online and includes a very quick and fair peer-review system, which is all easy to use. Visit http://www.dovepress.com/testimonials.php to read real quotes from published authors. 